\section{Ambiente, clases sociales y potencia emancipadora: contribuciones para una intervención social contrahegemónica desde los espacios académicos}

\author{
Martín Graziano \\ Universidad de Buenos Aires - Consejo \\ Nacional de Investigaciones Científicas \\ y Técnicas, Argentina \\ marting@ege.fcen.uba.ar \\ (iD) orcid.org/0000-0002-6876-7211
}

Ambiente y extensión universitaria /

Perspectivas

다(1)(2)
RECEPCIÓN: 30/06/21

ACEPTACIÓN FINAL: 20/09/21

\section{Resumen}

Los espacios académicos poseen un amplio espectro de intervención en el tejido social en vinculación con colectivos socioambientales, escuelas y otras organizaciones sociales, en un tiempo actual atravesado por la urgencia de una crisis ecológica en ciernes. Argumentaré aquí que la constitución de alianzas contrahegemónicas entre la academia y diversos movimientos sociopolíticos de la clase trabajadora, en particular los movimientos sociales territoriales que agrupan a trabajadoras y trabajadores desocupados o marginados de la economía formal, permiten la intervención a través de la coproducción de conocimiento crítico al sistema y la implementación de prácticas ecosociales prefigurativas ligadas a la acción sociopolítica necesaria y conducente al cambio de sistema. Este tipo de alianzas abona a una práctica científica y académica que persiga el fortalecimiento de potencialidades emancipadoras en organizaciones sociopolíticas autónomas de la clase trabajadora, aspecto cada vez más necesario para revertir las crisis por venir.

Palabras clave: coproducción de conocimiento; marxismo; alianzas contrahegemónicas; extensión crítica movimientos sociales territoriales.
Environment, social classes and emancipatory power: contributions for a counterhegemonic social intervention from academic spaces

\section{Abstract}

Academic spaces have a wide spectrum of intervention in the social fabric in connection with socio-environmental groups, schools and other social organizations, in a current time crossed by the urgency of a looming ecological crisis. I will argue here that the constitution of counter-hegemonic alliances between academia and various socio-political movements of the working class, in particular territorial social movements that bring together unemployed or marginalized workers from the formal economy, allow intervention through the co-production of knowledge critical to the system and the implementation of pre-figurative eco-social practices, in connection with the necessary socio-political action conducive to system change. This type of alliance supports a scientific and academic practice that seeks to strengthen emancipatory potentialities in autonomous socio-political organizations of the working class, an aspect that is increasingly necessary to reverse the crises to come.

Keywords: knowledge co-production; marxism; counter-hegemonic alliances; critical academy; territorial social movements.
Meio ambiente, classes sociais e poder emancipatório: contribuições para uma intervenção social contra-hegemônica a partir dos espaços acadêmicos

\section{Resumo}

Os espaços acadêmicos têm amplo espectro de intervenção no tecido social em articulação com grupos socioambientais, escolas e outras organizações sociais, em um momento atual atravessado pela urgência de uma iminente crise ecológica. Argumentarei aqui que a constituição de alianças contra-hegemônicas entre a academia e diversos movimentos sociopolíticos da classe trabalhadora, em particular os movimentos sociais territoriais que reúnem trabalhadores desempregados ou marginalizados da economia formal, permitem a intervenção por meio da coprodução de conhecimento crítico para o sistema e a implementação de práticas ecossociais prefigurativas, em conexão com a ação sociopolítica necessária e conducente à mudança do sistema. Esse tipo de aliança sustenta uma prática científica e acadêmica que busca fortalecer as potencialidades emancipatórias nas organizações sociopolíticas autônomas da classe trabalhadora, aspecto cada vez mais necessário para reverter as crises que estão por vir.

\section{Palavras-chave: coprodução de} conhecimento; marxismo; alianças contrahegemônicas; extensão crítica; movimentos sociais territoriais

Para citación de este artículo: Graziano, M. (2021). Ambiente, clases sociales y potencia emancipadora: contribuciones para una intervención social contrahegemónica desde los espacios académicos. +E: Revista de Extensión Universitaria, 11(15), e0001. doi: 10.14409/extension.2021.15.Jul-Dic.e0001 


\section{Introducción}

Vivimos tiempos con un ritmo acelerado de degradación ambiental que impacta notablemente sobre la calidad de vida de distintos sectores del pueblo y las comunidades. Esta degradación se manifiesta en forma de contaminación del agua, del suelo o del aire, de destrucción del hábitat natural, de pérdida de biodiversidad o de complejidad en las tramas de la vida. A nivel global, diversos trabajos académicos dan cuenta de los diferentes límites ecológicos planetarios que hemos cruzado o estamos en riesgo de cruzar (Rockström et al., 2009; Barnosky et al., 2012), como, por ejemplo, el umbral de temperatura para que el sistema Tierra continúe siendo un sumidero de carbono (Duffy et al., 2021). Esta y otras situaciones configuran el aspecto biofísico de la crisis ecológica en ciernes.

Ahora bien, detrás de este escenario podemos visualizar una compleja interacción de distintas crisis desplegadas en el sistema capitalista en su fase actual de desarrollo. Según Nancy Fraser, destacada contribuyente a la teoría social crítica, vivimos una crisis multidimensional del capitalismo que integra una pluralidad de conflictos sociales - democráticos, ambientales, de género y etnia- desde una perspectiva marxista (Fraser, 2020). Estas "luchas de frontera" (Fraser, 2015) tienen el potencial de ampliar el sujeto revolucionario a otros ámbitos por fuera de la tradición marxista clásica, pero también pueden convertirse en luchas absorbidas por el sistema a partir de la concesión de reformas en su interior que no varían sustancialmente las relaciones de opresión y explotación (Valdivielso Navarro, 2019).

En tanto, si bien para David Harvey (2014) las crisis son esenciales para la reproducción del sistema capitalista, la relación del capital con la naturaleza es una de esas "contradicciones peligrosas" que atraviesa al sistema dada su plausibilidad de socavar su motor de funcionamiento, la vida humana misma, y de abrir la posibilidad de que exista un límite objetivo para la expropiación de la naturaleza. La interacción entre el sistema de producción capitalista y la crisis ecológica hoy se ve fácilmente explicitada en las causas estructurales de la pandemia, que pueden atribuirse a características específicas de la fase actual del capitalismo asociada a la concentración de la renta agrícola, la persistencia de procesos de acumulación por despojo, y la necesidad de importantes niveles de consumo globalizados (Wallace et al., 2020; Díaz et al., 2020). Así, cuando se afirma con liviandad que la salida a la crisis ecológica va a ser resultado del esfuerzo y responsabilidad de la humanidad en su conjunto, muy a tono con una perspectiva posmoderna, es necesario agudizar la crítica y la imaginación para ofrecer diagnósticos e intervenciones radicalmente alternativas.

En este contexto, las preguntas que quisiera abordar en este trabajo se relacionan con los elementos que puedan dar cuenta del rol de las clases sociales en la crisis ecológica, acerca de cómo unificar esa lucha de frontera con una perspectiva de clase y a partir de ahí reconstruir la estrategia para intervenir social y políticamente desde los espacios académicos a través de una salida superadora del sistema capitalista. ¿Qué relación existe entre las clases sociales y la degradación ambiental? ¿Qué capas sociales van a llevar a cabo los cambios necesarios para una transformación radical del sistema? Y teniendo en cuenta esto, ¿cómo abordar la intervención académica en el territorio con relación a los conflictos socioambientales? ¿Qué estrategia darnos como trabajadores de la ciencia y la educación para la transformación contrahegemónica?

Voy a argumentar aquí que la constitución de alianzas contrahegemónicas entre la academia crítica, aquella que es capaz de reconocer el fracaso e inequidad de un modo de 
producción y consumo que nos ha llevado a una profunda crisis socioecológica, y diversos movimientos sociopolíticos de la clase trabajadora, en particular sectores de los distintos movimientos sociales territoriales que agrupan a trabajadoras y trabajadores marginados de la economía formal, permiten la intervención social mediante la coproducción de conocimiento crítico al sistema y la implementación de prácticas ecosociales prefigurativas en vinculación con la acción sociopolítica necesaria y conducente al cambio de sistema. Para ello, tomo la definición de políticas prefigurativas de la larga tradición anarquista y autonomista (Graeber, 2002; Ouviña, 2013), implementada por una gran mayoría de los movimientos populares territoriales de hoy en Argentina y otras partes de Latinoamérica, y que aspiran en su lucha por un cambio social profundo a producir transformaciones en sus propias prácticas y modos de vida presente que prefiguren la nueva sociedad a la que se pretende llegar (Ouviña, 2013). Resalto finalmente una consideración fundamental de lo antedicho y en general olvidada en favor de alguno de los polos de atracción: ambos factores (la coproducción/ prefiguración y la acción sociopolítica) deben ir en conjunto para que las alianzas propuestas tengan potencia emancipadora colectiva o de masas (Farber, 2014).

\section{Ambiente y clases sociales en el capitalismo actual}

La profunda interrelación entre los modos de apropiación de la naturaleza y el sistema de producción y consumo capitalista remarcado por diversos autores (Moore, 2020; Malm, 2020, Fraser, 2021) llevan a preguntarnos acerca del rol de las clases sociales dentro de esta crisis civilizatoria. Tomo la definición de clase social de Nicos Poulantzas, uno de los principales teóricos marxistas del siglo XX, para quien "las clases sociales son conjuntos de agentes sociales que se diferencian principal, pero no exclusivamente, por su lugar en el proceso de producción" (Poulantzas, 2016, pp. 12-13), incluyendo también y de manera relevante elementos de subordinación-dominación ideológicos y políticos que hacen a la determinación de clase. Podemos distinguir entre las clases fundamentales a la burguesía (dominante del sistema capitalista) y a la clase obrera e incorporar a la pequeña burguesía (tanto la tradicional —pequeños propietarios - como a la nueva pequeña burguesía, es decir, trabajadores de servicios comerciales y públicos, como empleados de oficina, negocios, instituciones del Estado, caracterizada por el trabajo no productivo) como polarizada entre dichas clases fundamentales (Poulantzas, 2016). A su vez, esas clases se encuentran divididas en fracciones o capas de clase que pueden tener diferentes posicionamientos al respecto más allá de su determinación estructural.

Aclaradas algunas definiciones conceptuales necesarias para el análisis posterior, considero que podemos distinguir una doble asimetría en cuanto a la interacción entre las clases sociales y el acceso/apropiación del ambiente o la naturaleza.

Por un lado, existe abundante evidencia acerca del impacto desigual que ejerce la clase dominante sobre la naturaleza a partir de la predominancia de formas de apropiación de la misma para el trabajo productivo, como la deforestación a gran escala, la agricultura y ganadería industrial, el uso de energías fósiles y la megaminería, entre otras (Martínez-Alier, 2005; Boyce, 1994). Un ejemplo de esto es la apropiación por parte de la burguesía terrateniente de tecnologías intensivas que degradan el ambiente, en contraposición a formas alternativas de producción implementadas por sectores de pequeños productores o comunidades 
indígenas (Gras y Cáceres, 2020). A nivel global, la teoría del intercambio ecológico desigual intenta explicar los patrones también desiguales de daño ambiental a raíz de la división internacional del trabajo: las naciones del Norte Global, más ricas y poderosas, tienen un acceso desproporcionado a los recursos naturales, y las naciones del Sur Global son las receptoras de los desechos generados por ello (Givens et al., 2019).

Además, podemos mencionar un acceso desigual a los beneficios ecosistémicos que provee la naturaleza para la calidad de vida de las personas (Berbés-Blázquez et al., 2016; Ernstson, 2013). Esto es, la existencia de una apropiación desigual de estos beneficios entre la clase dominante y el resto de las clases evidenciada, por ejemplo, en el acceso a una mejor calidad de agua para consumo, a servicios recreativos, al disfrute de la naturaleza (ríos y arroyos urbanos con alta integridad ecológica), y a un entorno habitacional con mayor biodiversidad, entre otros elementos (Schell et al., 2020).

Esas asimetrías suponen que tanto la falta de acceso a los beneficios de la naturaleza para el ser humano como la degradación ambiental afectan predominantemente a algunas fracciones de la pequeña burguesía y a la clase obrera menos calificada, incluyendo las capas desocupadas o marginadas del trabajo. En nuestro país en particular, la pobreza y la desocupación son una característica estructural de los últimos 40 años y tienen estrecha relación con la falta de acceso a agua potable, a áreas verdes o cuerpos de agua con alta biodiversidad e integridad ecológica, a la residencia en áreas frecuentemente inundables o con contaminación de las aguas superficiales, el suelo y el aire, por mencionar solo algunos ejemplos (Tobías y Fernández, 2019; Ríos 2015; Graziano et al., 2021).

El análisis precedente intenta esclarecer el carácter de clase en lo que respecta al impacto sobre la naturaleza y el hábitat natural y el acceso a dicha naturaleza en cuanto a los beneficios sociales, culturales y económicos, y pone en perspectiva la necesidad de su comprensión para que una intervención desde los espacios académicos tenga potencia contrahegemónica. Esto no implica que sectores de la pequeña burguesía, campesinos o la misma clase obrera en su trabajo productivo o reproductivo no ejerzan prácticas degradantes o de apropiación local de la naturaleza —véase, por ejemplo, Fraser (2021) sobre la apropiación de la naturaleza bajo el socialismo-. En el marco de su teoría ampliada del capitalismo, Fraser (2020) argumenta que este último aspecto, más que debilitar la relación entre expropiación de la naturaleza y sociedad de clases, representaría las consecuencias de una de las condiciones de posibilidad del capitalismo (sus "moradas ocultas"), es decir, la escisión histórica y cualitativamente inédita entre sociedad y naturaleza alcanzada desde el surgimiento del capitalismo y la era moderna (Nahuel Martin, 2020). ${ }^{1}$

En la próxima sección analizaremos desde qué posición social las investigadoras y los investigadores inciden en este contexto para luego referirnos a las vías por las cuales es necesario avanzar desde el marco de una práctica crítica de la actividad científica.

\section{El Estado, las y los intelectuales y la necesidad de un posicionamiento de clase}

Si partimos de la concepción del Estado desarrollada por Poulantzas (2005), el Estado capitalista monopolista actual es la condensación material de las relaciones de fuerza entre 
clases y fracciones de clase, atravesado en su conjunto por los intereses tácticos y estratégicos de la burguesía o clase dominante. Para Poulantzas, el Estado no es refugio de una clase $o$ un instrumento que se puede utilizar hacia un lado $u$ otro, sino el terreno donde se materializan los antagonismos de clase bajo relaciones de dominación (Thwaites Rey, 2007).

Desde esta perspectiva, tanto los espacios académicos como los espacios de investigación público-estatales, como parte de los aparatos de Estado, reflejan esos antagonismos de clase bajo la dominación ideológica y política de la clase dominante. Dicho de otra manera, la enseñanza superior pública y estatal, en tanto parte constituyente de los aparatos ideológicos del Estado, no escapa a la función de dominación y reproducción del sistema capitalista, pero se encuentra condicionada por las alianzas de clases del bloque en el poder (Poulantzas, 2016). Y atento a los determinantes estructurales de clase relativos al lugar ocupado dentro del modo de producción capitalista, las investigadoras, los investigadores y docentes de enseñanza superior formamos parte de lo que Poulantzas denominó la "nueva pequeña burguesía", que posee como características fundamentales la falta de un programa político autónomo y se encuentra polarizada por las clases fundamentales (la clase obrera y la burguesía). La precarización y burocratización del trabajo, sumadas a su baja jerarquía en lo que hace a los roles de vigilancia y dominación dentro de las relaciones de producción, hacen a esta fracción de la pequeña burguesía más cercana a la clase obrera que a la clase dominante.

Antonio Gramsci (2000), por su parte, fue un pionero en remarcar la necesidad de que la clase obrera tenga sus propios intelectuales orgánicos como instrumento fundamental para la construcción contrahegemónica. Esto no tiene que entenderse como una participación necesaria dentro de las organizaciones políticas autónomas de la clase trabajadora (a lo cual, sin embargo, adhiero), pero sí como tener un recorrido en común y un papel importante en virtud de su participación activa en la vida práctica, es decir, en la acción, a la hora de construir una nueva cultura, una nueva hegemonía (Pulleiro, 2013).

Esta caracterización conlleva, a mi entender, dos consecuencias para nuestro quehacer cotidiano que pueden aplicarse a las diferentes esferas de trabajo académico:

1) El posicionamiento de clase de las y los intelectuales es fundamental para la acción contrahegemónica si lo que pretendemos es un cambio de sistema que ponga en discusión los modos de producción y consumo actual, y no solo hacer mejoras en el sistema existente (léase el "no solo" como algo no excluyente ni contrapuesto a promover reformas en el sistema actual).

2) La necesidad de generar alianzas de clase con otros sectores de la clase trabajadora que permitan coproducir conocimiento crítico y experiencias prefigurativas donde se constituyan otras relaciones sociales y para con la naturaleza, a la vez que se incremente la potencia emancipadora de la clase como tal (construcción de una fuerza social revolucionaria).

Aquí quizás es donde podemos empezar a vislumbrar algunas intersecciones entre una política de alianzas contrahegemónicas y las múltiples dimensiones de ruptura de la institucionalidad capitalista. Retomando la caracterización realizada en la introducción, una de las consecuencias de la crisis multidimensional del capitalismo en su fase neoliberal es la aparición de luchas de frontera (racial, de género, democráticas, y ambientales, por supuesto) que en la manera en que se desplegaron desde una cosmovisión posmoderna no han 
podido visualizar la interacción de dichas luchas. Tras la ofensiva neoliberal que impuso grandes derrotas a la clase obrera en todo el mundo, la ideología dominante buscó separar la lucha de los movimientos sociales, como el ecologista, de las demandas de la clase trabajadora. Pierre Bourdieu, en su artículo "Para un saber comprometido" (2002), se refería a la necesidad de alianzas de los sectores académicos con otros sectores de la clase trabajado$\mathrm{ra}$, como los sindicatos y los nuevos movimientos sociales. Afirmaba que las investigadoras y los investigadores debían "favorecer la aparición de condiciones organizacionales para la producción colectiva de la intención de inventar un proyecto político" y las condiciones para su éxito. Proporcionar instrumentos, ayudar a inventar y fortalecer nuevos rumbos políticos a través de un saber comprometido y romper a la vez la falsa dicotomía entre academia y compromiso. Por su parte, Orlando Fals Borda, en su texto "Irrumpe la investigación militante", menciona que "el trabajo realizado de esta forma, con trabajadores urbanos y campesinos está demostrando la utilidad de esta orientación para producir no solo una ciencia fresca y propia, sino hechos políticos que llevan a transformar radicalmente nuestra sociedad" (2013, p. 208).

A continuación, voy a argumentar que, siguiendo los desarrollos teóricos realizados anteriormente, la construcción de un sujeto social ampliado que se apropie de las luchas ecológicas y sociales de manera holística para una transformación radical del sistema se verá favorecida por una alianza de clases con los movimientos sociales territoriales que organizan a las capas de trabajadoras y trabajadores más precarizados y vulnerados, las y los que sufren las inequidades sociales y ecológicas del sistema. Esta es a mí entender la estrategia más adecuada en la coyuntura actual para intervenir desde los espacios académicos con una perspectiva contrahegemónica. ${ }^{2}$

\section{Movimientos sociales territoriales: entre la prefiguración ecosocial y la potencia antisistémica}

Decíamos antes que la degradación ambiental afecta más predominantemente a fracciones de la pequeña burguesía, como trabajadores de servicios comerciales y públicos (empleados de oficina, negocios, instituciones del Estado) y a la clase obrera menos calificada, incluyendo la población desocupada y marginada del trabajo. A su vez, mencionábamos la necesidad de generar alianzas de clases en donde las investigadoras, los investigadores, académicas y académicos puedan desplegar un posicionamiento de clase en tanto intelectual orgánico, y proponíamos que los movimientos sociales territoriales sirvieran de lugar estratégico para dichas alianzas en la constitución de un sujeto social revolucionario ampliado. Ahora bien, ¿a qué nos referimos con movimientos sociales territoriales?

Las lógicas neoliberales de los años 90 en Argentina trastocaron no solo las funciones del Estado sino la vida misma de las trabajadoras y los trabajadores. Las privatizaciones, las

2) Desde ya, pueden pensarse e incluso analizarse como válidas alianzas con otras fracciones de la clase obrera en el marco de sus organizaciones políticas (sindicatos, partidos). Si bien tengo argumentos para justificar una elección sobre otra en cuanto a la potencia emancipadora (ver más adelante), también puedo brindar argumentos para la generación de alianzas del sector académico con otras fracciones de la clase obrera (como, por ejemplo, el posicionamiento estratégico de diferentes sectores de la clase en la producción y circulación de mercancías). A este respecto ver la última sección del artículo. 
leyes de flexibilización laboral, la corrupción pública, entre otras cuestiones, reconfiguraron las lógicas del capital con efectos devastadores para buena parte de la clase obrera y de la pequeña burguesía, tanto económica como socialmente (Svampa y Pereyra, 2003). Las instituciones barriales comunitarias tendieron a su desaparición o descomposición en el tejido local (sociedades de fomento, clubes de barrio), la desocupación y precarización pasaron a ser moneda corriente, y a partir de allí fueron surgiendo nuevos movimientos sociales con marcada territorialidad para luchar por distintas mejoras en las condiciones de vida (trabajo genuino, salud, educación, tierra y vivienda) pero también para disputar en el terreno de la acción política desde una perspectiva, en muchos casos, antisistémica (Svampa y Pereyra, 2003; Pacheco, 2010). Ejemplos de ello fueron los distintos Movimientos de Trabajadores Desocupados (MTDs) y el Movimiento Teresa Rodríguez (MTR), e incluso algunos dieron lugar a las organizaciones sociales actuales, como el Frente de Organizaciones en Lucha (FOL) y el Frente Popular Darío Santillán en sus diferentes vertientes actuales, por mencionar solo dos. Durante la década del 90 surgieron asimismo movimientos campesinos como el MOCASE, que luego dio lugar al Movimiento Nacional Campesino Indígena $(\mathrm{MNCl})$ a raíz del avance de la frontera agrícola con el consecuente desplazamiento de comunidades, campesinas y campesinos de sus tierras, y que ha conllevado a diversas prácticas contrahegemónicas en articulación con sectores críticos de la academia (Desalvo, 2015; García et al., 2021). Por último, podríamos incluir aquí, con algunas salvedades respecto de la heterogénea fracción de clase organizada, a las asambleas ambientales que aparecieron a lo largo y ancho de nuestro territorio en la disputa contra el extractivismo minero y otras formas de explotación y apropiación de la naturaleza (Svampa y Antonelli, 2009; Merlinsky, 2013). Muchas de ellas han podido establecer diferentes niveles de organización regional y nacional con capacidad de acción política.

Estos movimientos sociales territoriales, en la medida en que no sean cooptados por la clase dominante, juegan un rol fundamental al momento de establecer alianzas de clase para la superación de la crisis ecológica por su potencia emancipadora, en tanto principales sujetos afectados simultáneamente por la apropiación y degradación del ambiente por parte de las clases dominantes y su condición de fracción de clase marginalizada del trabajo productivo formal. Como mencionamos, este aspecto se entrelaza con una teoría ampliada del capitalismo, donde se destaca no solo la necesidad de modificación de la propiedad de los medios de producción sino todo un entramado de condiciones de posibilidad del capitalismo, ampliando la concepción de sujeto revolucionario hacia otras esferas de opresión.

Así, la conjugación de tres aspectos fundamentales al interior de estos movimientos, como son la preeminencia de prácticas prefigurativas en el territorio, la capacidad de movilización, y la acción política como parte de la lucha de clases (Ouviña, 2013), favorece la potencia emancipadora de esta alianza de clases. Las prácticas ecosociales prefigurativas tienen la capacidad de antagonizar los paradigmas vigentes de destrucción y degradación del ambiente y revalorizar el contacto cotidiano con la naturaleza, el cuidado y recuperación de los beneficios ecosistémicos que los ambientes naturales proveen (culturales, espirituales, de soporte y provisión de cuidados). En la siguiente sección me referiré al despliegue de estas potencialidades ecosociales prefigurativas de manera sinérgica con la academia crítica. 


\section{Forma y contenido de las alianzas académico-territoriales}

Quiero destacar dos aspectos que hacen al despliegue de dichas articulaciones o alianzas desde la perspectiva académica en coproducción con el territorio:

En primer lugar, desde mi visión, la articulación académico-territorial puede darse en torno a dos modos de acción contrahegemónicos y ligados a la acción política transformadora: i) la coproducción de experiencias locales y regionales que prefiguren modos alternativos de producción y reproducción de la vida, ii) la coproducción de conocimiento crítico antisistema. En cualquiera de las modalidades, la coproducción implica la ruptura de las lógicas del saber experto neutral y la socialización del mismo así como el quiebre de su despolitización, que se evite además la distinción del trabajo intelectual/trabajo manual y se incorpore a ese proceso, por ejemplo, la toma de decisiones colectivas como parte fundamental (Turnhout et al., 2020). Existen hoy en día distintos paradigmas desarrollados dentro de la investigación y la extensión universitaria desde los cuales puede desarrollarse esta perspectiva, como la transdisciplina, entendida desde un enfoque coproductivo (Stokols, 2006; Lang et al., 2012, Graziano, 2019), la Investigación-Acción Participativa (IAP) (Keahey, 2021; Fals Borda, 2013), y la extensión crítica (Tommasino y Cano, 2016). La creación de espacios intersectoriales en el territorio dirigidas por los propios movimientos sociales son puntos de partida importantes en este sentido, y existen diversas iniciativas tanto en el ámbito rural como en el urbano (Cruz-Sousa et al., 2011; García et al., 2021; Graziano, 2019).

Algunas temáticas de intervenciones socioambientales que se pueden promover a partir de alianzas académico-territoriales con los movimientos sociales son: el fortalecimiento de huertas urbanas y periurbanas, la implementación de prácticas agroecológicas, la constitución y capacitación de cuadrillas ambientales de trabajo territorial para una mejora del hábitat, la coproducción de conocimiento social crítico referido a la contaminación de las aguas para consumo humano, la implementación de cuadrillas de reciclado ambiental y recuperación de materiales, la recuperación y mantenimiento de espacios verdes públicos, el establecimiento de viveros de plantas nativas y su reintroducción al entorno, el mantenimiento de arroyos urbanos desde un manejo comunitario y ecológico de los mismos, tareas de promoción ambiental y prevención de enfermedades transmitidas por mosquitos, etc. En muchos de estos casos existe a su vez un gran potencial en la capacidad de autogestión e impacto sobre el trabajo en ramas productivas y de servicios. En general, las experiencias llevadas a cabo con movimientos sociales urbanos y rurales poseen distintos desafíos por delante y limitaciones en lo que respecta a su potencia emancipadora (Graziano, 2019; Graziano et al., 2019; Lanzarotti et al., 2016; Di Matteo et al., 2021; Paladino et al., 2020). ${ }^{3}$

En segundo lugar, considero que la potencia emancipadora de la articulación académico-territorial se encuentra asociada al grado de organización sociopolítica conseguida y su horizonte de cambio social. Con esto quiero decir que la intervención social puede tener diferente potencial emancipador según el tipo de articulación y el posicionamiento de clase logrado, ya sea con colectivos de vecinas y vecinos afectados por un determinado conflicto ambiental, organizaciones comunitarias territoriales sin articulación regional, o con

3) No quiero entrar en detalle aquí porque sería extenderme en una evaluación que necesita un análisis más pormenorizado, pero considero que existe muchas veces una tensión entre el despliegue de la potencia prefigurativa y el foco sobre la acción política antisistémica, mediada fundamentalmente por las diferentes concepciones políticas y las vías para la lucha de clases. 
organizaciones socioterritoriales articuladas regionalmente con otros sectores de la clase trabajadora. A mi entender, el máximo potencial emancipador se encuentra bajo articulaciones sociopolíticas con organizaciones que promuevan tanto la acción política antisistémica como la prefiguración de experiencias alternativas con horizonte ecosocial.

Pero este planteo no se encuentra exento de tensiones al interior de la academia, e incluso dentro de los sectores críticos de los paradigmas productivos vigentes y su impacto ambiental negativo, ya que aún hoy persiste la noción de "neutralidad" en la producción del conocimiento científico, conjugada con caracterizaciones mayormente implícitas en cuanto al Estado y su función en nuestra sociedad (Gurvich et al., 2009; Silvetti et al., 2013). Esto tiene su corolario en el despliegue de la práctica científica bajo una determinada "independencia" respecto de la acción política, con predominio, en el mejor de los casos, de un enfoque "proveedor" de conocimiento para la adopción de políticas públicas estatales que, en su mayoría, nunca llegan ( $\mathrm{y}$, dado el carácter de clase que hemos discutido anteriormente, nunca van a llegar). Este último enfoque es muy marcado en el ámbito de la ecología (Gurvich et al., 2009).

\section{Algunas reflexiones finales}

Para finalizar, y a modo de síntesis y recapitulación, quería dejar remarcadas algunas ideas que fueron recorridas en este trabajo y que nos pueden permitir seguir reflexionando sobre vías alternativas o formas de profundizar lo expuesto hasta aquí:

- Partimos de considerar "lo ambiental" como un eje de intervención en el marco de una teoría ampliada del capitalismo donde la intervención contrahegemónica pasa hoy, en esta fase del capitalismo, por el feminismo, el ecologismo, y la reversión de las desigualdades y opresiones de etnia, clase, género.

- En este marco, distinguimos una doble asimetría en cuanto a la interacción entre las clases sociales y el ambiente:

a) No todas las clases sociales afectan por igual al ambiente y a la naturaleza, y dimos evidencia de cómo las clases dominantes impactan en el ambiente en mayor medida que las clases explotadas.

b) No todas las clases sociales acceden de igual manera a los beneficios que pueden proveer los ecosistemas, lo que evidencia las disparidades en el acceso entre las clases dominantes y la clase trabajadora.

- Esta interacción entre clase y ambiente posibilita comprender la crisis ecológica y su "lucha de frontera" como parte de un entramado de relaciones desplegadas por el modo de producción y consumo capitalista, donde se hace necesario la unificación de las luchas por las distintas opresiones subyacentes y articularlas en un sujeto revolucionario ampliado.

- La degradación ambiental, al afectar más predominantemente a la clase obrera menos calificada y por sobre todo a los sectores de trabajadores desocupados y marginados del trabajo productivo, sumada a la potencia sociopolítica antisistema y prefigurativa de un sector de los movimientos territoriales actuales, me lleva a proponer una alianza contrahegemónica en el terreno de la articulación académico-territorial que fortalezca la unidad de esas luchas de frontera (ecológicas, feministas, étnicas) desde una perspectiva clasista. 
- Esta alianza académico-territorial, desplegada como un conjunto de prácticas coproductivas, requiere de un posicionamiento de clase de las trabajadoras y los trabajadores de la investigación y la docencia en universidades que haga factible su construcción como intelectual orgánico, operando como articulador en una dimensión técnico-política bajo la orientación de las organizaciones sociopolíticas mencionadas anteriormente.

El marco de alianza propuesto con las organizaciones sociales antisistémicas de la clase obrera marginada del trabajo formal implica desde ya una apuesta a un nuevo sujeto social que pueda conjugar las distintas luchas ante las múltiples crisis institucionales del capitalismo actual. Los crecientes niveles de flexibilización laboral, la marginación de un sector cada vez más grande de la clase trabajadora, y el impacto en las próximas décadas de las nuevas revoluciones tecnológicas y del cambio climático global (migraciones forzadas, formación de guetos urbanos) sobre la precarización de la vida, no hacen más que abonar a la creciente necesidad de su organización política para una transformación radical del sistema. Claro que es posible proponer otras alianzas contrahegemónicas con distintas fracciones de la clase trabajadora organizadas en sindicatos de base, corrientes sociales y políticas, o partidos políticos que representen los intereses de la clase trabajadora (Huber, 2021). Sin lugar a dudas, el horizonte a construir es una amplia alianza con organizaciones sociopolíticas que nucleen a las diferentes capas subalternas y a las tendencias políticas anticapitalistas, sin sectarismos ni hegemonismos, en pos de unificar las luchas desplegadas desde las distintas aristas del capitalismo actual.

En lo que a nosotras y nosotros respecta como trabajadores de la ciencia y la educación, es indispensable seguir abonando por una práctica científica y académica que persiga el fortalecimiento de potencialidades emancipadoras en organizaciones sociopolíticas autónomas de la clase trabajadora, bajo un sistema social donde la tecnociencia es un factor predominante en la expansión del capital y se hace cada vez más necesaria una intervención eficaz para revertir las crisis por venir.

\section{Referencias bibliográficas}

Barnosky, A. D.; Hadly, E. A.; Bascompte, J.; Berlow, E. L.; Brown, J. H.; Fortelius, M.; Getz, W. M.; Harte, J.; Hastings, A.; Marquet, P.; Martinez, N. D.; Mooers, A.; Roopnarine, G.V.; Williams, J.W.; Gillespie, R.; Kitzes, J.; Marshall, C.; Matzke, N.; Mindell, D. P. (...) Smith A. B. (2012). Approaching a state shift in Earth's biosphere. Nature, 486(7401), 52.

Berbés-Blázquez, M.; González, J. A. y Pascual, U. (2016). Towards an ecosystem services approach that addresses social power relations. Current Opinion in Environmental Sustainability, 19, 134-143.

Bourdieu, P. (2002). Por un saber comprometido. Le Monde Diplomatique. https://www. bloghemia.com/2021/05/ para-un-saber-comprometido-por-pierre.html

Boyce, J. K. (1994). Inequality as a cause of environmental degradation. Ecological economics, 11(3), 169-178. Cruz-Souza, F.; Cortegoso, A. L.; Zanin, M. y Shimbo, I. (2011). Las incubadoras universitarias de economía solidaria en Brasil: un estudio de casos. Revista de estudios cooperativos, (106), 74-94.

Desalvo, A. (2015). Las acciones en defensa de la tierra en Santiago del Estero (1990-2012). El caso del MOCASE. Eutopia: Revista de Desarrollo Económico Territorial, 8, 57-74.

Di Matteo, Á. J.; Palumbo, M. M.; Plaza, B. L. y Almada, A. (2021). Consideraciones acerca de la producción 
audiovisual en la investigación compartida junto con movimientos populares. Masquedós-Revista de Extensión Universitaria, 6(6), 13-23.

Díaz, S.; Cáceres, D. M.; León, A. E.; Presman, C.; Bernardello, G. y Perillo, M (2020). La pandemia COVID-19 es el resultado del modelo de apropiación de la naturaleza. En Solanet, M. A. (Ed.). Pandemia: los desafíos múltiples que en el presente le plantea al porvenir. Academia Nacional de Ciencias Morales y Políticas.

Duffy, K. A.; Schwalm, C. R.; Arcus, V. L.; Koch, G. W.; Liang, L. L. y Schipper, L. A. (2021). How close are we to the temperature tipping point of the terrestrial biosphere? Science advances, 7(3), eaay1052.

Ernstson, H. (2013). The social production of ecosystem services: A framework for studying environmental justice and ecological complexity in urbanized landscapes. Landscape and urban planning, 109(1), 7-17.

Fals Borda, O. (2013). Ciencia, compromiso y cambio social. El Colectivo.

Farber, S. (2014). Reflexiones sobre la política prefigurativa. Nueva Sociedad, (251), 70.

Fraser, N. (2015). Legitimation crisis? On the political contradictions of financialized capitalism. Critical Historical Studies, 2(2), 157-189.

(2020). Los talleres ocultos del capital. Un mapa para la izquierda. Traficantes de Sueños.

(2021). Climates of capital. For a Trans-Environmental Eco-Socialism. New Left Review, (127), 94-127.

García, M.; Figueroa, A. E.; Villar, S. y Calefato, N. (2021). Movimientos sociales, agroecología y soberanía alimentaria. Un acercamiento al proyecto pedagógico de la Universidad Campesina-Sistemas Universitarios Rurales Indoamericanos, Santiago del Estero, Argentina. + E: Revista de Extensión Universitaria, 11(14), 1-21.

Givens, J. E.; Huang, X. y Jorgenson, A. K. (2019). Ecologically unequal exchange: A theory of global environmental injustice. Sociology Compass, 13(5), e12693.

Gurvich, D. E.; Renison, D. y Barri, F. (2009). El rol del ecólogo ante la crisis ambiental actual. Ecología austral, 19(3), 233-238.

Graeber, D. (2002). Los nuevos anarquistas. New Left Review, 13, 139-151.

Gramsci, A. (2000). Los intelectuales y la organización de la cultura. Nueva Visión.

Gras, C. y Cáceres, D. M. (2020). Technology, nature's appropriation and capital accumulation in modern agriculture. Current Opinion in Environmental Sustainability, 45, 1-9.

Graziano (2019). Ciencia y Territorio: co-generando caminos para la transformación social. En Cortelezzi, A.; Entraigas, I.; Grosman, F.; Masson, I (Eds.). Encuentro de Saberes para la gestión responsable de ecosistemas acuáticos pampeanos. Buenos Aires.

Graziano, M.; De Groot, G.; Pilato, L.; Sánchez, M.; Izaguirre, I. y Pizarro, H. (2019). Fostering urban transformations in Latin America: lessons around the ecological management of an urban stream in coproduction with a social movement (Buenos Aires, Argentina). Ecology and Society, 24(4).

Graziano, M.; Giorgi, A. y Feijoó, C. (2021). Multiple stressors and social-ecological traps in Pampean streams (Argentina): a conceptual model. Science of the Total Environment, 765, 142785.

Harvey, D. (2014). Diecisiete contradicciones y el fin del capitalismo. Traficantes de Sueños.

Huber, M. (2021). Una política ecológica para la clase trabajadora. Jacobin América Latina. https://jacobinlat. com/2021/05/29/10041/.

Keahey, J. (2021). Sustainable development and participatory action research: a systematic review. Systemic Practice and Action Research, 34(3), 291-306.

Lang, D. J.; Wiek, A.; Bergmann, M.; Stauffacher, M.; Martens, P.; Moll, P.; Swilling, M. y Thomas, C. J. (2012). Transdisciplinary research in sustainability science: practice, principles, and challenges. Sustainability science, 7(1), 25-43.

Lanzarotti, E. O.; Cuesta, G.; Factorovich, M. H.; Kucher, H.; Prudkin Silva, C. R.; Lichtig, P.; Borón, I.; Álvarez, L.; Urdampilleta, C.; Vallerga, M. B.; Romero J. M.; Piegari, E.; Morzán, U.; De Groot, G.; Sirvan Baglietto, B.; 
Poveda Ducon, K. e Ithuralde, R. E. (2016). Tierra y agrotóxicos: un enfoque coproductivo en problemáticas socioambientales. Cambios y Permanencias, 7, 181-219.

Malm, A. (2020). Capital fósil. Traficantes de sueños.

Martínez-Alier, J. (2005). El ecologismo de los pobres: conflictos ambientales y lenguajes de valoración. Icaria. Merlinsky, G. (2013). Cartografías del conflicto ambiental en Argentina. 1ra. ed. CICCUS.

Moore, J. (2020). El capitalismo en la trama de la vida. Ecología y acumulación del capital. Traficantes de sueños.

Nahuel Martín, F. (2020). Nancy Fraser: de la distribución a la crítica del capitalismo. Diánoia, 65(85), 161-192. Ouviña, H. (2013). La política prefigurativa de los movimientos populares en América Latina. Hacia una nueva matriz de intelección para las Ciencias Sociales. Acta Sociológica, 62, 77-104.

Pacheco, M. (2010). De Cutral-Có a Puente Pueyrredón. Una genealogía de los Movimientos de Trabajadores Desocupados. El Colectivo.

Paladino, I. R.; Sokolowski, A. C.; Navas, M.; Gagey, M.; Barrios, M. B.; Rodríguez, H. A. y Wolski, J. E. (2020). Experiencia de extensión universitaria en huertas comunitarias de organizaciones barriales. Agrotecnia, 30, 5-16. Poulantzas, N. (2005). Estado, poder y socialismo. 9na. ed. Siglo XXI Editores. (2016). Las clases sociales en el capitalismo actual. 3ra. ed. Siglo XXI Editores.

Pulleiro, A. (2013). El papel de los intelectuales en la Argentina reciente: una aproximación a la experiencia de "Carta Abierta". Iberoforum. Revista de Ciencias Sociales de la Universidad Iberoamericana, VIII(15), 156-181.

Ríos, D. (2015). Present-day capitalist urbanization and unequal disaster risk production: the case of Tigre, Buenos Aires. Environment and Urbanization, 27(2), 679-692.

Rockström, J.; Steffen, W.; Noone, K.; Persson, Å.; Chapin III, F. S.; Lambin, E. F.; Lenton, T. M.; Scheffer, M.; Folke, C.; Schellnuber, H. J; Nykvist, B.; De Wit, C. A.; Hughes, T.; Van der Leeuw, S.; Rodhe, H.; Sorlin, S.; Snyder, P. K.; Costanza, R.; Svedin, U. (...); Foley, J. A. (2009). A safe operating space for humanity. Nature, 461(7263), 472.

Schell, C. J.; Dyson, K.; Fuentes, T. L.; Des Roches, S.; Harris, N. C.; Miller, D. S.; Woelfle-Erskine, C. A. y Lambert, M. R. (2020). The ecological and evolutionary consequences of systemic racism in urban environments. Science, 369(6510).

Silvetti, F.; Soto, G.; Cáceres, D. M. y Cabrol, D. (2013). ¿Por qué la legislación no protege los bosques nativos de Argentina? Conflictos socioambientales y políticas públicas. Mundo agrario, 13(26).

Stokols, D. (2006). Toward a science of transdisciplinary action research. American journal of community psychology, 38(1), 63-77.

Svampa, M. y Antonelli, M. (2009). Minería transnacional, narrativas del desarrollo y resistencias sociales. Biblos. Svampa, M. y Pereyra, S. (2003). Entre la ruta y el barrio. La experiencia de las organizaciones piqueteras. Biblos. Thwaites Rey, M. T. (2007). Complejidades de una paradójica polémica: estructuralismo versus instrumentalismo. En Thwaites Rey (Ed.). Estado y marxismo. Un siglo y medio de debates. Prometeo.

Tobías, M.; Fernández, L. (2019). La circulación del agua en Buenos Aires: resonancias geográficas y desigualdades socioespaciales en el acceso al servicio. Cuadernos de Geografía: Revista Colombiana de Geografía, 28(2), 423-441.

Tommasino, H. y Cano, A. (2016). Modelos de extensión universitaria en las universidades latinoamericanas en el siglo XXI: tendencias y controversias. Universidades, 67, 7-24. Unión de Universidades de América Latina y el Caribe.

Turnhout, E.; Metze, T.; Wyborn, C.; Klenk, N. y Louder, E. (2020). The politics of co-production: participation, power, and transformation. Current Opinion in Environmental Sustainability, 42, 15-21. 
Valdivielso Navarro, J. (2019). Sujetos contrahegemónicos y crisis del capitalismo según Nancy Fraser: ¿Se ha convertido el ecologismo en siervo del capitalismo? Eikasia: revista de filosofía, (89), 283-311.

Villanova, N. (2014). La organización política de los cartoneros en la ciudad de Buenos Aires: 1997-2012. Aportes para una caracterización de su desarrollo político. Cuadernos del CENDES, 31(87), 127-156.

Wallace, R.; Liebman, A.; Chaves, L. F. y Wallace, R. (2020). COVID-19 and Circuits of Capital. Monthly Review, 72(1), 1-13. 